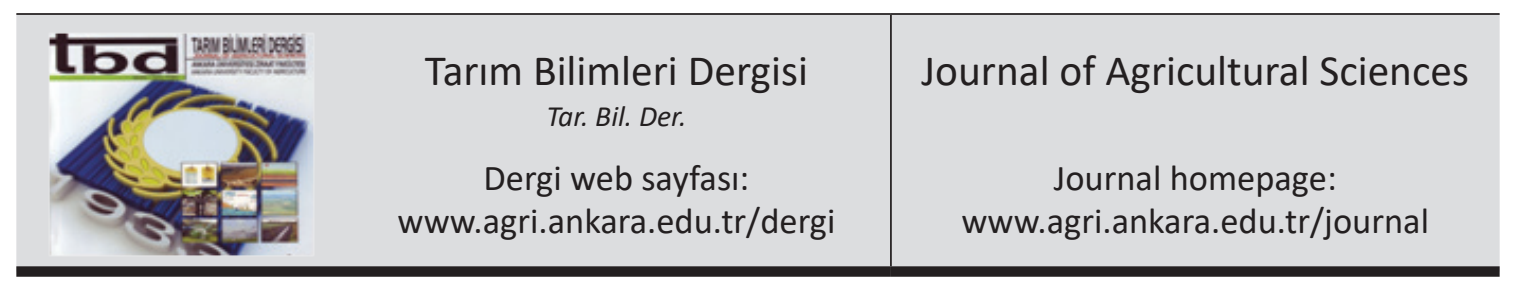

\title{
Characterization of Wild Apricot (Prunus armeniaca L.) Genotypes Selected from Cappadocia Region (Nevşehir-Turkey) by SSR Markers
}

\author{
Melike BAKIR ${ }^{\mathrm{a}}$, Hatice DUMANOĞLU ${ }^{\mathrm{b}}$, Veli ERDOĞAN ${ }^{\mathrm{b}}$, Cemil ERNIM'c ${ }^{\mathrm{c}}$, Tahir MACIT ${ }^{\mathrm{d}}$ \\ ${ }^{a}$ Erciyes University, Seyrani Faculty of Agriculture, Department of Agricultural Biotechnology, Kayseri, TURKEY \\ ${ }^{b}$ Ankara University, Faculty of Agriculture, Department of Horticulture, Ankara, 06110, TURKEY \\ ${ }^{c}$ Apricot Research Institute, Aşağıbağlar Mah. Ismetpaşa Caddesi, No: 163, Yeşilyurt, Malatya, TURKEY \\ ${ }^{d}$ Malatya Directorate of Provincial Agriculture and Forestry, Karakavak Mahallesi, Ankara Asfaltı, 7. km, Malatya, TURKEY
}

\section{ARTICLE INFO}

Research Article

Corresponding Author: Veli ERDOĞAN, E-mail: verdogan@agri.ankara.edu.tr, Tel: +90 (312) 5961256

Received: 06 September 2018, Received in Revised Form: 05 October 2018, Accepted: 31 October 2018

\begin{abstract}
Cappadocia region of Anatolia hosts the third largest wild apricot population in Turkey. The objective of the study was to characterize 44 wild apricot genotypes selected from Cappadocia Region (Nevşehir-Turkey) as prominent with their late flowering, resistance to spring late frosts, large fruit sizes and/or late fruit ripening characteristics and 5 reference apricot cultivars ('Hacıhaliloğlu', 'Kabaaşı', 'Hasanbey', 'Aprikoz' and 'Levent') with SSR (simple sequence repeats) markers. A total of 16 SSR primers were used and 13 of them were successfully amplified. Total number of alleles was 107, average number of alleles was 8.23; average $H e$ and $H o$ values were 0.722 and 0.669 , respectively. Polymorphism information content (PIC) values varied between 0.471 and 0.845 . There was a quite high genetic diversity among wild apricot genotypes that genetic similarity values varied between 12 and 96\%. Homonymous and synonymous genotypes were not encountered.
\end{abstract}

Keywords: Wild apricot; Prunus armeniaca; Genetic diversity; Genetic relationship; Molecular characterization; SSRs

(C) Ankara Üniversitesi Ziraat Fakültesi

\section{Introduction}

Vavilov (1951) indicated the origin centers of culture apricots (Prunus armeniaca L.) as China, Central Asia and defined Near-East centers extending from Northeastern Iran to Caucasus and Central Anatolia as the secondary origin center of cultured forms. Kostina (1969) divided P. armeniaca species into 4 large eco-geographical groups and 13 regional subgroups and placed Turkey into Iran-Caucasus ecogeographic group (Layne et al 1996; Zhebentyayeva et al 2012). Anatolia (Turkey) is located within the secondary origin center of apricots, thus has a great genetic diversity (Ercisli 2004). Nevşehir province is located right at the center of Cappadocia region of Anatolia, and the province hosts the $3^{\text {rd }}$ largest wild apricot population with about 145000 trees (TUIK 2017). This population is characterized with late flowering, resistance to spring late frosts, large fruits and late ripening. Thus the population exhibits a large variation in fruit physical and quality attributes. Such a diverse population was evaluated for the first time by Dumanoğlu et al (2018) within 
the scope of a scientific research project, and superior genotypes were identified. These genotypes were then put under protection in a collection orchard. The genotypes constitute significant materials for apricot breeding studies and genetic relationships among these genotypes should be identified with further molecular techniques. Microsatellites or simple sequence repeats (SSRs) are short repeat sequences (1-6 base length) and have co-dominant characteristics, greater polymorphism ratios, are abundant in genome and have quite high repeatability. Therefore, they have a significant place among DNA markers (Litt \& Luty 1989; Gupta et al 1996). These markers are commonly used in identification of species, preservation of genetic materials, population genetics, quantitative trait loci mapping, marker assisted selection and similar studies. SSR markers are also used in genetic characterization of Prunus species, including apricots. However, SSR markers were not developed at the same rates for each one of the significant species (apricot, peach, plum, and almond), thus potential use of SSR markers of a species in other Prunus species (crosstransferability) have become a significant issue (Hormaza 2002; Romero et al 2003; Zhebentyayeva et al 2003; Hagen et al 2004; Messina et al 2004; Mnejja et al 2005; Sanchez-Perez et al 2005; Ruthner et al 2006; Bouhadida et al 2009; Wünsch 2009; Akpınar et al 2010; Bourguiba et al 2010; Liu et al 2013; Wang et al 2014; Eroglu \& Cakir 2015; Gürcan et al 2015; Murathan et al 2017).

In this study, genetic relationships between wild apricot genotypes selected from the wild apricot gene sources of Nevşehir province with regard to late flowering, resistance to spring late frosts, large fruits and or late fruit ripening characteristics were identified with SSR markers developed from $P$. armeniaca and $P$. persica.

\section{Material and Methods}

\subsection{Plant material and DNA isolation}

In this study, 44 wild apricot genotypes selected from Nevşehir (Cappadocia Region-Turkey) locality and the reference apricot cultivars of
'Aprikoz', 'Kabaaşı', 'Hasanbey', 'Hacıhaliloğlu' and 'Levent' were used as the plant material. DNA isolations were performed from fresh shoot tips and young leaf samples collected from the genotypes (Lefort et al 1998). DNA purity and concentrations were determined in ND-1000 spectrophotometer and isolated DNA was visually controlled in $1 \%$ agarose gel.

\subsection{SSR reactions}

A total of 16 SSR loci were selected as of 10 P. armeniaca (apricot), 4 P. persica (peach) and $2 P$. armeniaca EST-SSR loci (Table 1). Selected SSR loci were tested and the polymorphic ones were used in genetic identifications. PCR amplifications were performed by using M13-tailed primer according to the methods described by Schuelke (2000) in Prunus genotypes. A tail (M13 universal sequence (-21), TGTAAAACGACGGCCAGT) was added to the 5' end of each forward primers. PCR amplifications were performed in $15 \mu \mathrm{L}$ reaction mixture containing $90 \mathrm{ng}$ genomic DNA, $0.1 \mu \mathrm{M}$ of each SSR primer, $0.1 \mu \mathrm{M}$ labelled M13 (-21) universal primer, $0.2 \mathrm{mM}$ of each dNTPs, $1 \mathrm{X}$ DreamTaq Green Buffer (includes $\mathrm{MgCl}_{2}$ at a concentration of $2 \mathrm{mM}$ ) (Thermo Scientific) and 0.5 U DreamTaq DNA Polymerase (Thermo Scientific). The amplification program consisted of an initial step of $3 \mathrm{~min}$ at $94{ }^{\circ} \mathrm{C}$, followed by 35 cycles of $1 \mathrm{~min}$ at $94{ }^{\circ} \mathrm{C}, 1 \mathrm{~min}$ at $50-66{ }^{\circ} \mathrm{C}, 2 \mathrm{~min}$ at $72{ }^{\circ} \mathrm{C}$, followed by 8 cycles of $1 \mathrm{~min}$ at $94{ }^{\circ} \mathrm{C}, 1 \mathrm{~min}$ at 53 ${ }^{\circ} \mathrm{C}, 2$ min at $72{ }^{\circ} \mathrm{C}$, and a final extension at $72{ }^{\circ} \mathrm{C}$ for $10 \mathrm{~min}$. The M13 (-21) primer was 5'-fluorescently tagged with HEX, 6-FAM or ROX to facilitate multiplexing. A set of three PCR products $(0.5 \mu \mathrm{L}$ each) was mixed with $0.5 \mu \mathrm{L}$ GeneScan-600 LIZ size standards (Applied Biosystems, USA) and $9.5 \mu \mathrm{L} \mathrm{Hi}-\mathrm{Di}^{\mathrm{TM}}$ formamide (Applied Biosystems) and denatured at $95{ }^{\circ} \mathrm{C}$ for $5 \mathrm{~min}$, chilled on ice and electrophoresed on the Applied Biosystems Prism 3500 Genetic Analyzer System (Applied Biosystems, USA). GENEMAPPER software v5.0 (Applied Biosystems, USA) was used to determine fragment size. 
Table 1- SSR markers used for characterization of wild apricot genotypes selected from Nevşehir locality

\begin{tabular}{|c|c|c|c|c|c|}
\hline No & Locus & $\begin{array}{l}\text { Primer sequences } \\
\left(5^{\prime} \rightarrow 3^{\prime}\right)\end{array}$ & $\begin{array}{l}\mathrm{Tm} \\
\left({ }^{\circ} \mathrm{C}\right)\end{array}$ & Species & Reference \\
\hline \multirow[t]{2}{*}{1} & UDAp-407F* & ttctgctacttacaatcgtgttctc & 56 & Prunus armeniaca & Messina et al (2004) \\
\hline & UDAp-407R & agagcaccaggtctttctgg & & & \\
\hline \multirow[t]{2}{*}{2} & UDAp-410F* & ttgttgacaagaagaaaacaaagc & 56 & Prunus armeniaca & Messina et al (2004) \\
\hline & UDAp-410R & caacgggttggtttcagaag & & & \\
\hline \multirow[t]{2}{*}{3} & UDAp-411F* & tcggtggagaaagagactgg & 56 & Prunus armeniaca & Messina et al (2004) \\
\hline & UDAp-411R & gtcccccaccetttacaatg & & & \\
\hline \multirow[t]{2}{*}{4} & UDAp-414F* & caagcacaagcgaacaaaat & 56 & Prunus armeniaca & Messina et al (2004) \\
\hline & UDAp-414R & ggtggtttcttatccgatgc & & & \\
\hline \multirow[t]{2}{*}{5} & UDAp-415F* & aactgatgagaaggggcttg & 56 & Prunus armeniaca & Messina et al (2004) \\
\hline & UDAp-415R & actcc gacatttgtgettc & & & \\
\hline \multirow[t]{2}{*}{6} & UDAp-418F* & cagaaatagccccagcacat & 56 & Prunus armeniaca & Messina et al (2004) \\
\hline & UDAp-418R & ttcttgcgccaaaaacaact & & & \\
\hline \multirow[t]{2}{*}{7} & UDAp-420F* & ttccttgettccettcattg & 56 & Prunus armeniaca & Messina et al (2004) \\
\hline & UDAp-420R & cccagaacttgattctgacca & & & \\
\hline \multirow[t]{2}{*}{8} & UDAp-423F* & ccatgtagaaactggetgagg & 56 & Prunus armeniaca & Messina et al (2004) \\
\hline & UDAp-423R & cactcgactctctcgectct & & & \\
\hline \multirow[t]{2}{*}{9} & AMPA105F* & ctgctctcactcaactcaatgc & 55 & Prunus armeniaca & Hagen et al (2004) \\
\hline & AMPA105R & ctccectaccectctgtatctc & & & \\
\hline \multirow[t]{2}{*}{10} & AMPA096F* & ttttgtgccaaagtagcatcag & 55 & Prunus armeniaca & Hagen et al (2004) \\
\hline & AMPA096R & tcaactaaccaaaaggagtggc & & & \\
\hline \multirow[t]{2}{*}{11} & UDP96-010F* & cccatgtgtgtccacatctc & 55 & Prunus persica & Cipriani et al (1999) \\
\hline & UDP96-010R & ttgatgattccatgcgtctc & & & \\
\hline \multirow[t]{2}{*}{12} & UDP96-019F* & ttggtcatgagctaagaaaaca & 55 & Prunus persica & Cipriani et al (1999) \\
\hline & UDP96-019R & tagtggcacagagcaacacc & & & \\
\hline \multirow[t]{2}{*}{13} & UDP98-406F * & tcggaaactggtagtatgaacaga & 55 & Prunus persica & Cipriani et al (1999) \\
\hline & UDP98-406R & atgggtcgtatgcacagtca & & & \\
\hline \multirow[t]{3}{*}{14} & ${\mathrm{Ma} 040 \mathrm{aF}^{*}}$ & agaaattggagtgacgtaac & 55 & Prunus persica & Yamamoto et al (2002) \\
\hline & $\mathrm{Ma} 040 \mathrm{aR}$ & acgtgatgagaagtagggag & & & \\
\hline & EST-SSR Primers & tcggaaactggtagtatgaacaga & 55 & Prunus persica & Cipriani et al (1999) \\
\hline \multirow[t]{2}{*}{15} & AMPA116F* & attgaaggcccettatgtgag & 55 & Prunus armeniaca-EST & Hagen et al (2004) \\
\hline & AMPA116R & caaaaaggcgttacagatgatg & & & \\
\hline \multirow[t]{2}{*}{16} & AMPA119F* & gtgeccacttacctgttttagg & 55 & Prunus armeniaca-EST & Hagen et al (2004) \\
\hline & AMPA119R & tcgacgatcagacttgctacag & & & \\
\hline
\end{tabular}

*, Fluorescent labeled primer

\subsection{Statistical analysis}

For each locus, the expected heterozygosity $(\mathrm{He})$, observed heterozygosity $(\mathrm{Ho})$ and polymorphism information content (PIC) (Nei 1973) were calculated with PowerMarker V3.025 software (Liu \& Muse 2005). The neighbor-joining (NJ) and unweighted pair-group method using arithmetic average (UPGMA) were used to construct and draw a dendrogram from the genetic similarity matrix by using the MEGA6 (Tamura et al 2007) and PowerMarker software programs. Bootstrap analyses with 100 replicates were performed and a consensus tree was obtained to measure the confidence levels for the clusters. 


\section{Results}

Allele sizes (bp, base-pair) for 13 SSR loci of wild apricot types selected from Nevşehir locality and reference apricot cultivars are provided in Table 2. Considering the success ratios of 16 SSR loci selected for genetic characterization of wild apricot types, 10-15 of them were thought to be used in genetic analyses. Thirteen SSR loci were successfully amplified. Of the remaining 3 SSR loci, UDP98-406 locus yielded successful PCR reactions, but was not able to be assessed well because of mixed peaks in capillary electrophoresis system. While PCR reactions of AMPA096 locus were unsuccessful, UDP96-019 locus was identified as monomorphic. Therefore, these 3 loci were not used in genetic analyses. The highest number of alleles was observed in UDAp-418 locus with 14 alleles (Table 3). It was followed by both UDP96010 and AMPA105 loci with 10 alleles and the lowest number of alleles was observed in $\mathrm{Ma} 040$ locus with 5 alleles. Total number of alleles was 107 and average number of alleles was 8.23 . Average $\mathrm{He}$ and $\mathrm{Ho}$ values were calculated 0.722 and 0.669 , respectively. $H e$ values varied between 0.501 and 0.860 and $H o$ values varied between 0.449 and 0.816. Polymorphism information content (PIC) value varied between 0.471 (AMPA119) and 0.845 (UDP96-010) (Table 3).

Genetic similarity dendrogram indicated that genotypes were basically separated into 3 groups (Figure 1). When the dendrogram was evaluated based on the places from where the samples were collected, it was observed that grouping was independent from the sampling locations. Considering the place of reference apricot cultivars in the dendrogram, 'Hasanbey' was placed in the second group, and the other cultivars were placed in $3^{\text {rd }}$ group. Reference cultivars were not placed in the first group.

Genetic similarity index values varied between 12 and $96 \%$ with the greatest similarity (96\%) between wild apricot genotypes of \#6 and \#61. These genotypes were followed by the \#32-\#34 and $\# 39-\# 68$ with a similarity ratio of $92 \%$. The lowest genetic similarity $(12 \%)$ was observed between the genotypes \#13 and \#38, \#38 and \#50,\#38 and \#64, and \#45 and \#47. Degree of genetic similarity was independent from the sampling locations. Genotypes \#6 and \#61, $45 \mathrm{~km}$ away from each other (Çavuşin and Gümüşkent, respectively) had the highest genetic similarity $(96 \%)$ in the study. However, more distantly located $(>65 \mathrm{~km})$ genotypes \#39 (Gümüşkent) and \#68 (Çakıllı), or genotypes \#32 and \#34 at the same location (Yeşilöz) had the same genetic similarity level of $92 \%$.

\section{Discussion}

Hormaza (2002) used 37 SSR loci developed from Prunus species to identify the genetic relationships among 48 apricot genotypes collected from different geographical regions. Of these loci, 31 were successfully amplified, 20 had repeatable polymorphic characteristics and a total of 82 alleles were identified in 48 genotypes. The common primer UDP96-100 was also found to be polymorphic in this study. Polymorphic UDP98-406 primer was not able to be assessed because of complex peak profile in our study. In another study, Romero et al (2003) used 16 SSR loci developed from peach genome to identify the relationships among 40 apricot genotypes collected from different eco-geographical regions. Of these loci, 11 presented polymorphism in apricot genotypes and allowed clear identification of each genotype. The common primer UDP96-010 similarly yielded the greatest separation power in this study. Zhebentyayeva et al (2003) tested 30 SSR loci developed through enriched library method from peach genome for 74 apricot genotypes. Of 30 SSR loci tested, 20 were amplified in apricot and 14 were reported to be used in separation of apricot genotypes and apricot germplasm diversity studies. Messina et al (2004) isolated 99 SSR loci from apricots and tested 20 of them in 16 apricot genotypes to determine polymorphism ratios. Of 20 SSR loci tested, 9 (UDAp-401, UDAp-404, UDAp-407, UDAp-410, UDAp-411, UDAp-414, UDAp-415, UDAp-418 and UDAp-420) were recommended to be used in apricot fingerprinting studies. Researchers also implied that $20 \%$ of 
Table 2- Allelic data (bp) for wild apricot genotypes of Nevşehir locality and standard apricot cultivars (A, 'Aprikoz'; L, 'Levent'; HB, 'Hasanbey'; HH, 'Hacihaliloğlu'; KA, 'Kabaaşı')

\begin{tabular}{|c|c|c|c|c|c|c|c|c|c|c|c|c|}
\hline \multirow{2}{*}{$\frac{\text { Genotype \# }}{3}$} & \multicolumn{2}{|c|}{$U D A P 411$} & \multicolumn{2}{|c|}{$U D A P 415$} & \multicolumn{2}{|c|}{$\mathrm{Ma040}$} & \multicolumn{2}{|c|}{ AMPA116 } & \multicolumn{2}{|c|}{$U D A P 414$} & \multicolumn{2}{|c|}{ UDAP423 } \\
\hline & 84 & 110 & 163 & 175 & 225 & 225 & 135 & 165 & 166 & 178 & 184 & 198 \\
\hline 4 & 88 & 110 & 163 & 175 & 233 & 233 & 135 & 135 & 166 & 186 & 184 & 206 \\
\hline 6 & 110 & 110 & 169 & 179 & 223 & 241 & 135 & 137 & 166 & 186 & 184 & 184 \\
\hline 7 & 88 & 110 & 169 & 179 & 223 & 223 & 135 & 137 & 178 & 190 & 184 & 204 \\
\hline 13 & 88 & 88 & 169 & 169 & 223 & 223 & 135 & 165 & 166 & 166 & 184 & 198 \\
\hline 14 & 84 & 110 & 175 & 179 & 223 & 233 & 135 & 135 & 178 & 186 & 184 & 204 \\
\hline 15 & 88 & 110 & 169 & 177 & 223 & 241 & 135 & 165 & 166 & 186 & 184 & 204 \\
\hline 16 & 84 & 84 & 169 & 169 & 223 & 223 & 135 & 135 & 178 & 186 & 184 & 204 \\
\hline 17 & 110 & 110 & 163 & 175 & 223 & 223 & 135 & 135 & 178 & 178 & 190 & 206 \\
\hline 18 & 110 & 110 & 163 & 169 & 223 & 233 & 135 & 135 & 166 & 178 & 184 & 206 \\
\hline 19 & 88 & 88 & 169 & 175 & 233 & 241 & 135 & 159 & 178 & 186 & 184 & 190 \\
\hline 20 & 110 & 110 & 169 & 179 & 223 & 241 & 135 & 137 & 166 & 186 & 184 & 184 \\
\hline 21 & 110 & 110 & 163 & 163 & 233 & 233 & 135 & 153 & 166 & 180 & 180 & 204 \\
\hline 22 & 110 & 110 & 169 & 169 & 233 & 241 & 135 & 135 & 186 & 186 & 184 & 204 \\
\hline 23 & 110 & 122 & 163 & 163 & 223 & 225 & 165 & 165 & 166 & 178 & 184 & 206 \\
\hline 24 & 110 & 122 & 167 & 167 & 223 & 233 & 135 & 153 & 178 & 178 & 198 & 204 \\
\hline 26 & 84 & 109 & 163 & 163 & 223 & 241 & 135 & 153 & 178 & 186 & 198 & 206 \\
\hline 27 & 110 & 110 & 163 & 169 & 223 & 223 & 135 & 159 & 178 & 186 & 184 & 206 \\
\hline 28 & 104 & 104 & 167 & 179 & 223 & 233 & 135 & 137 & 166 & 178 & 184 & 204 \\
\hline 29 & 110 & 122 & 169 & 175 & 223 & 223 & 135 & 137 & 178 & 190 & 184 & 184 \\
\hline 31 & 88 & 122 & 163 & 167 & 223 & 223 & 135 & 165 & 166 & 166 & 184 & 198 \\
\hline 32 & 110 & 110 & 169 & 175 & 223 & 223 & 135 & 153 & 179 & 179 & 184 & 198 \\
\hline 33 & 110 & 122 & 179 & 179 & 223 & 233 & 135 & 159 & 166 & 178 & 184 & 200 \\
\hline 34 & 110 & 110 & 169 & 175 & 223 & 223 & 135 & 153 & 178 & 178 & 184 & 198 \\
\hline 35 & 110 & 110 & 169 & 179 & 223 & 233 & 135 & 135 & 178 & 186 & 184 & 184 \\
\hline 38 & 110 & 110 & 175 & 181 & 233 & 233 & 145 & 145 & 178 & 178 & 184 & 184 \\
\hline 39 & 84 & 84 & 169 & 175 & 233 & 233 & 135 & 135 & 178 & 178 & 184 & 184 \\
\hline 41 & 104 & 110 & 175 & 175 & 223 & 223 & 135 & 135 & 166 & 178 & 184 & 204 \\
\hline 42 & 88 & 88 & 167 & 175 & 223 & 223 & 135 & 135 & 178 & 186 & 184 & 200 \\
\hline 43 & 110 & 110 & 167 & 175 & 223 & 233 & 135 & 159 & 166 & 186 & 184 & 204 \\
\hline 45 & 110 & 110 & 163 & 179 & 223 & 233 & 135 & 135 & 166 & 178 & 184 & 200 \\
\hline 46 & 84 & 110 & 163 & 179 & 233 & 241 & 135 & 165 & 166 & 178 & 184 & 204 \\
\hline 47 & 88 & 88 & 175 & 175 & 223 & 223 & 135 & 166 & 166 & 166 & 198 & 204 \\
\hline 48 & 104 & 104 & 169 & 175 & 223 & 223 & 135 & 137 & 186 & 186 & 186 & 199 \\
\hline 49 & 88 & 110 & 163 & 167 & 223 & 223 & 135 & 153 & 178 & 178 & 184 & 204 \\
\hline 50 & 84 & 109 & 163 & 163 & 223 & 233 & 135 & 135 & 166 & 186 & 184 & 206 \\
\hline 53 & 84 & 104 & 169 & 175 & 223 & 233 & 135 & 137 & 179 & 186 & 186 & 206 \\
\hline 54 & 88 & 110 & 169 & 175 & 225 & 241 & 135 & 165 & 166 & 186 & 190 & 204 \\
\hline 59 & 110 & 122 & 163 & 169 & 223 & 223 & 159 & 165 & 166 & 186 & 184 & 204 \\
\hline 60 & 88 & 110 & 175 & 175 & 223 & 233 & 135 & 135 & 178 & 186 & 184 & 204 \\
\hline 61 & 110 & 110 & 169 & 179 & 223 & 241 & 135 & 137 & 166 & 186 & 184 & 184 \\
\hline 64 & 88 & 110 & 169 & 169 & 223 & 223 & 129 & 135 & 184 & 184 & 184 & 200 \\
\hline 68 & 84 & 84 & 169 & 175 & 233 & 233 & 135 & 135 & 178 & 178 & 184 & 184 \\
\hline 76 & 88 & 88 & 163 & 175 & 233 & 233 & 135 & 165 & 166 & 186 & 184 & 206 \\
\hline A & 110 & 110 & 163 & 169 & 223 & 225 & 129 & 159 & 184 & 184 & 184 & 198 \\
\hline HB & 84 & 84 & 175 & 181 & 223 & 233 & 135 & 143 & 166 & 180 & 180 & 198 \\
\hline $\mathrm{HH}$ & 84 & 84 & 167 & 175 & 223 & 223 & 135 & 153 & 180 & 180 & 180 & 204 \\
\hline $\mathrm{KA}$ & 84 & 110 & 163 & 167 & 223 & 223 & 135 & 153 & 180 & 186 & 184 & 204 \\
\hline $\mathrm{L}$ & 84 & 110 & 167 & 167 & 223 & 241 & 135 & 153 & 180 & 186 & 204 & 204 \\
\hline
\end{tabular}


Table 2 (Continue)- Allelic data (bp) for wild apricot genotypes of Nevşehir locality and standard apricot cultivars (A, 'Aprikoz'; L, 'Levent'; HB, 'Hasanbey'; HH, 'Hacıhaliloğlu'; KA, 'Kabaaşı')

\begin{tabular}{|c|c|c|c|c|c|c|c|c|c|c|c|c|c|c|}
\hline \multirow{2}{*}{$\frac{\text { Genotype \# }}{3}$} & \multicolumn{2}{|c|}{ UDAP96-010 } & \multicolumn{2}{|c|}{ UDAP418 } & \multicolumn{2}{|c|}{ AMPA105 } & \multicolumn{2}{|c|}{ AMP119 } & \multicolumn{2}{|c|}{$U D A P 420$} & \multicolumn{2}{|c|}{$U D P 407$} & \multicolumn{2}{|c|}{$U D P 410$} \\
\hline & 93 & 117 & 176 & 180 & 203 & 203 & 114 & 122 & 195 & 195 & 204 & 206 & 142 & 162 \\
\hline 4 & 93 & 99 & 180 & 180 & 195 & 195 & 120 & 122 & 195 & 195 & 186 & 206 & 140 & 168 \\
\hline 6 & 113 & 117 & 176 & 182 & 203 & 203 & 116 & 120 & 179 & 195 & 204 & 204 & 140 & 142 \\
\hline 7 & 99 & 105 & 154 & 170 & 205 & 205 & 116 & 120 & 195 & 195 & 186 & 204 & 166 & 166 \\
\hline 13 & 117 & 117 & 170 & 170 & 205 & 231 & 120 & 124 & 179 & 179 & 186 & 204 & 142 & 142 \\
\hline 14 & 105 & 117 & 176 & 180 & 227 & 227 & 120 & 130 & 179 & 195 & 182 & 204 & 136 & 166 \\
\hline 15 & 93 & 99 & 176 & 180 & 203 & 205 & 116 & 120 & 169 & 195 & 186 & 204 & 142 & 142 \\
\hline 16 & 113 & 113 & 170 & 180 & 231 & 231 & 116 & 122 & 195 & 195 & 186 & 208 & 162 & 166 \\
\hline 17 & 99 & 103 & 180 & 180 & 227 & 231 & 120 & 120 & 169 & 195 & 186 & 204 & 136 & 168 \\
\hline 18 & 93 & 103 & 150 & 180 & 195 & 231 & 120 & 120 & 169 & 171 & 186 & 206 & 142 & 168 \\
\hline 19 & 97 & 117 & 180 & 180 & 195 & 205 & 116 & 122 & 179 & 195 & 182 & 186 & 140 & 140 \\
\hline 20 & 114 & 117 & 176 & 184 & 203 & 203 & 120 & 120 & 179 & 195 & 204 & 204 & 140 & 142 \\
\hline 21 & 99 & 113 & 150 & 152 & 203 & 227 & 120 & 120 & 169 & 195 & 186 & 186 & 136 & 136 \\
\hline 22 & 103 & 105 & 176 & 180 & 195 & 217 & 120 & 120 & 179 & 195 & 186 & 204 & 140 & 162 \\
\hline 23 & 113 & 117 & 150 & 180 & 195 & 227 & 116 & 120 & 169 & 195 & 186 & 204 & 140 & 168 \\
\hline 24 & 105 & 117 & 170 & 180 & 195 & 227 & 116 & 120 & 179 & 195 & 204 & 209 & 140 & 162 \\
\hline 26 & 93 & 117 & 152 & 176 & 217 & 231 & 120 & 120 & 169 & 179 & 186 & 206 & 140 & 168 \\
\hline 27 & 99 & 103 & 176 & 176 & 205 & 217 & 120 & 120 & 189 & 195 & 206 & 206 & 140 & 166 \\
\hline 28 & 93 & 113 & 174 & 180 & 195 & 195 & 120 & 120 & 171 & 195 & 182 & 186 & 136 & 142 \\
\hline 29 & 91 & 117 & 176 & 180 & 205 & 227 & 120 & 122 & 195 & 195 & 186 & 208 & 162 & 162 \\
\hline 31 & 103 & 117 & 180 & 180 & 205 & 227 & 120 & 120 & 171 & 195 & 204 & 204 & 142 & 168 \\
\hline 32 & 103 & 117 & 176 & 180 & 195 & 195 & 120 & 120 & 169 & 195 & 182 & 206 & 140 & 162 \\
\hline 33 & 99 & 105 & 182 & 182 & 195 & 205 & 120 & 120 & 169 & 195 & 182 & 206 & 136 & 142 \\
\hline 34 & 103 & 117 & 176 & 180 & 195 & 195 & 120 & 120 & 169 & 195 & 182 & 206 & 140 & 162 \\
\hline 35 & 97 & 105 & 176 & 180 & 205 & 227 & 116 & 120 & 187 & 195 & 186 & 186 & 162 & 168 \\
\hline 38 & 97 & 97 & 170 & 180 & 205 & 205 & 129 & 129 & 195 & 195 & 209 & 209 & 144 & 144 \\
\hline 39 & 105 & 105 & 154 & 164 & 195 & 195 & 120 & 120 & 195 & 195 & 186 & 209 & 140 & 162 \\
\hline 41 & 93 & 93 & 176 & 180 & 227 & 231 & 120 & 130 & 183 & 195 & 186 & 186 & 142 & 166 \\
\hline 42 & 105 & 117 & 176 & 180 & 195 & 227 & 120 & 120 & 171 & 179 & 186 & 204 & 142 & 162 \\
\hline 43 & 97 & 99 & 176 & 176 & 195 & 205 & 116 & 120 & 179 & 195 & 182 & 186 & 142 & 166 \\
\hline 45 & 97 & 97 & 150 & 150 & 226 & 226 & 120 & 130 & 171 & 195 & 182 & 182 & 140 & 168 \\
\hline 46 & 93 & 97 & 154 & 180 & 195 & 195 & 120 & 120 & 169 & 195 & 186 & 186 & 140 & 166 \\
\hline 47 & 103 & 117 & 164 & 176 & 205 & 231 & 116 & 122 & 169 & 169 & 186 & 208 & 142 & 166 \\
\hline 48 & 91 & 117 & 150 & 180 & 227 & 231 & 120 & 122 & 169 & 171 & 186 & 206 & 166 & 166 \\
\hline 49 & 91 & 105 & 152 & 176 & 203 & 227 & 120 & 120 & 169 & 185 & 186 & 186 & 166 & 166 \\
\hline 50 & 97 & 117 & 176 & 181 & 195 & 231 & 120 & 122 & 169 & 195 & 184 & 186 & 140 & 162 \\
\hline $53 *$ & 105 & 117 & 150 & $\begin{array}{l}170 \\
180\end{array}$ & 205 & $\begin{array}{l}217 \\
227\end{array}$ & 120 & 120 & 169 & 195 & 186 & 206 & 136 & 162 \\
\hline 54 & 97 & 99 & 154 & 176 & 195 & 205 & 120 & 120 & 169 & 195 & 204 & 204 & 142 & 168 \\
\hline 59 & 97 & 105 & 176 & 176 & 195 & 205 & 120 & 120 & 169 & 169 & 182 & 186 & 136 & 168 \\
\hline 60 & 105 & 113 & 154 & 184 & 195 & 205 & 116 & 120 & 183 & 195 & 186 & 204 & 140 & 140 \\
\hline 61 & 113 & 117 & 176 & 182 & 203 & 231 & 116 & 120 & 179 & 195 & 204 & 204 & 140 & 142 \\
\hline 64 & 113 & 113 & 170 & 170 & 191 & 191 & 120 & 120 & 169 & 171 & 186 & 204 & 136 & 140 \\
\hline 68 & 105 & 105 & 154 & 154 & 195 & 195 & 120 & 122 & 195 & 195 & 186 & 209 & 140 & 162 \\
\hline 76 & 93 & 99 & 164 & 182 & 195 & 195 & 120 & 122 & 195 & 195 & 186 & 206 & 140 & 168 \\
\hline A & 105 & 113 & 169 & 176 & 227 & 227 & 120 & 122 & 169 & 195 & 186 & 205 & 162 & 162 \\
\hline HB & 97 & 105 & 164 & 182 & 195 & 205 & 116 & 120 & 169 & 195 & 186 & 206 & 142 & 168 \\
\hline HH & 99 & 105 & 164 & 180 & 205 & 205 & 120 & 120 & 185 & 195 & 204 & 206 & 140 & 168 \\
\hline KA & 105 & 105 & 162 & 164 & 205 & 227 & 120 & 120 & 171 & 195 & 204 & 206 & 140 & 142 \\
\hline $\mathrm{L}$ & 99 & 103 & 148 & 180 & 227 & 227 & 120 & 120 & 169 & 185 & 186 & 204 & 142 & 168 \\
\hline
\end{tabular}

*, Genotype \#53 has 3 alleles and shows triploidy for the loci of UDAP418 and AMPA105 
Table 3- Genetic parameters for wild apricot genotypes of Nevşehir Locality (number of alleles (n), expected (He) and observed $(\mathrm{Ho})$ heterozygosity, polymorphism information content, PIC)

\begin{tabular}{lllll}
\hline Locus & $n$ & $H e$ & $H o$ & $P I C$ \\
\hline UDAp-411 & 6 & 0.675 & 0.449 & 0.634 \\
UDAp-415 & 7 & 0.787 & 0.714 & 0.755 \\
Ma040 & 5 & 0.601 & 0.469 & 0.546 \\
AMPA116 & 9 & 0.607 & 0.673 & 0.585 \\
UDAp-414 & 7 & 0.751 & 0.673 & 0.710 \\
UDAp-423 & 9 & 0.715 & 0.816 & 0.684 \\
UDP96-010 & 10 & 0.860 & 0.796 & 0.845 \\
UDAp-418 & 14 & 0.821 & 0.755 & 0.801 \\
AMPA105 & 10 & 0.812 & 0.571 & 0.788 \\
AMPA119 & 7 & 0.501 & 0.510 & 0.471 \\
UDAp-420 & 8 & 0.682 & 0.755 & 0.641 \\
UDAp-407 & 8 & 0.747 & 0.735 & 0.710 \\
UDAp-410 & 7 & 0.825 & 0.776 & 0.801 \\
\hline Total & 107 & 9.385 & 8.694 & 8.971 \\
Average & 8.23 & 0.722 & 0.669 & 0.690 \\
\hline
\end{tabular}

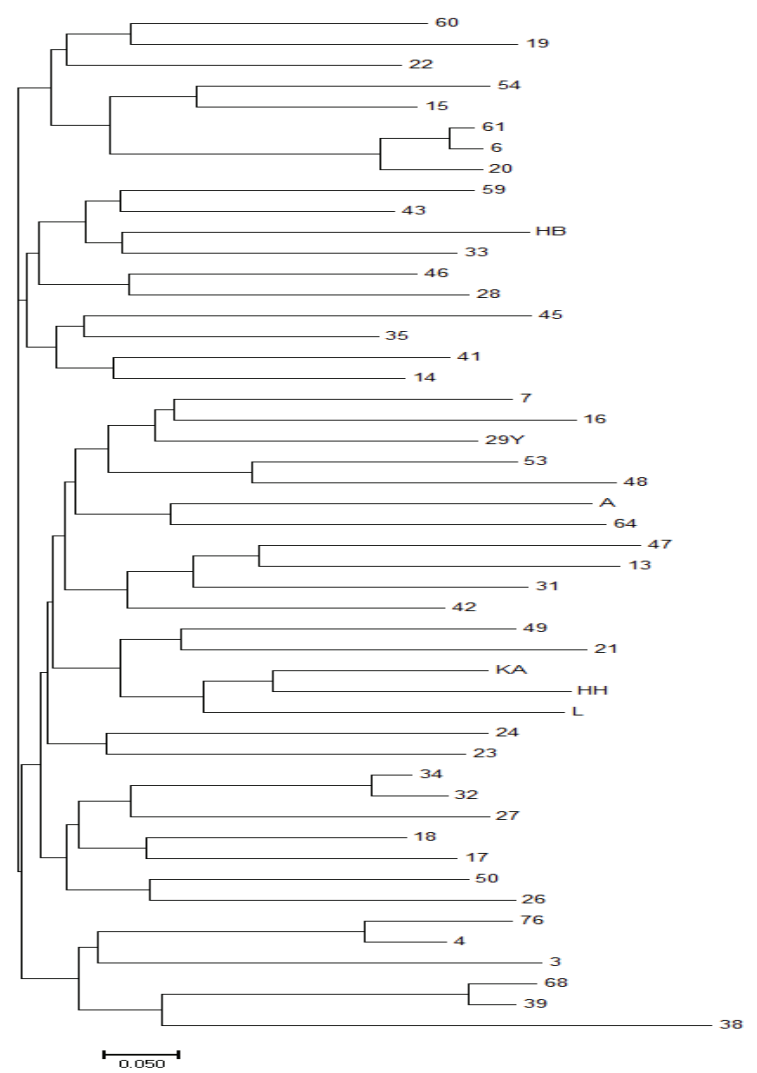

Figure 1- UPGMA (Unweighted pair group method with arithmetic average) based dendrogram showing genetic similarity between 44 wild apricot genotypes of Nevşehir locality and 5 reference apricot cultivars (A, Aprikoz; L, Levent; HB, Hasanbey; HH, Hacıhaliloğlu; KA, Kabaaşı) based on 13 SSR loci 
SSR loci were successfully amplified in different Prunus species including peaches, nectarine, almond, European plum, Japanese plum, cherry and sour cherry. Of the relevant primers, UDAp401 and UDAp-404 were also used in the present study and successfully amplified and had quite high polymorphism ratios. Akpınar et al (2010) used 10 SSR loci of which 2 were developed from apricots and 8 from peaches for genetic identification of 25 local apricot genotypes and 4 foreign reference cultivars. In the present study, the primer UDP96010 developed from the peaches had also similar polymorphisim. Ullah et al (2017) employed 20 SSR loci for genetic characterization of 12 apricot genotypes significant for the economy of Pakistan and reported polymorphic bands for 18 of them.

Zhebentyayeva et al (2003) characterized 74 apricot genotypes with 30 SSR markers and reported the number of alleles between 2 (Pchgms 17) and 16 (UPD 96-001) and total number of alleles as 107 with an average number of alleles as 7.64. Messina et al (2004) tested 20 of 99 SSR loci isolated from apricots in 16 apricot genotypes to determine polymorphism ratios and reported the number of alleles between 2 and 9, expected heterozygosity between 0.26 and 0.82 . Liu et al (2013) developed 19 microsatellite loci for marker assisted selection (MAS) of P. sibirica L. with regard to late flowering and characterized them in 40 genotypes. Researchers reported the number of alleles between 3 and 11, expected and observed heterozygosity ratios between 0.063 and 0.917 , and between 0.295 and 0.876 , respectively. In another study carried out with $P$. sibirica L. species, 31 SSR loci were used to assess genetic diversity and population structure. The number of alleles were reported between 5 and 33 with an average value of 19.323, and average expected and observed heterozygosity ratios were reported 0.639 and 0.774 , respectively (Wang et al 2014). Gürcan et al (2015) assessed 278 apricot genotypes with 20 SSR loci and reported the number of alleles between 5 and 25 with an average number of alleles as 12.78 , expected and observed heterozygosity values of 0.75 and 0.63 , respectively. Study, 49 P. armeniaca genotypes including wild apricot genotypes and reference cultivars were screened through 13 SSR markers and total number of alleles was identified as 107 , average number of alleles per locus as 8.23 , polymorphism information content as 0.69 .

SSR markers are used for various purposes (genetic mapping and etc.), especially for genetic characterization of Prunus species, including apricots. However, SSR markers were not able to be developed at the same rates for each one of the significant species (apricot, peach, plum, almond), thus potential use of SSR markers of a species in other Prunus species (cross-transferability) have become a significant issue. In this study, two different sources were preferred in selection of SSR loci for genetic assessment of wild apricot genotypes. Of these loci, while only one of 10 SSR loci (AMPA096) was unsuccessful, peak quality was poor in UDP98-406 locus developed from P. persica, and the UDP96019 locus developed from the same source was monomorphic. EST-SSR primers developed from $P$. armeniaca were successfully amplified and identified as polymorphic.

\section{Conclusions}

In this study, 16 SSR primers developed from apricot and peaches were used. Thirteen SSR primers were successfully amplified, and they had quite high polymorphism ratios. These 13 SSR loci were found sufficient and successful for characterization and identification of selected wild apricot genotypes. There was a quite high genetic diversity among the genotypes. Genetic similarity varied between 12 and $96 \%$, and homonymous and synonymous genotypes were not encountered.

\section{Acknowledgements}

This research was supported by TUBITAK (The Scientific and Technological Research Council of Turkey), project no 1140279. Assistance of Nevşehir Directorate of Provincial Food Agriculture and Forestry in field studies, and Assoc. Prof. Dr. Zeki GOKALP for comments and suggestions for the manuscript were greatly appreciated. 


\section{References}

Akpınar A E, Koçal H, Ergül A, Kazan K, Selli M E, Bakır M, Aslantas Ş, Kaymak S \& Sarıbas R (2010). SSRbased molecular analysis of economically important Turkish apricot cultivars. Genetics and Molecular Research 9: 324-332

Bouhadida M, Casas A M, Gonzalo M J, Arus P, Morenoa M A \& Gogorcena Y (2009). Molecular characterization and genetic diversity of Prunus rootstocks. Scientia Horticulturae 120: 237-245

Bourguiba H, Khadari B, Krichen L, Trifi-Farah N, Santoni S \& Audergon J M (2010). Grafting versus seed propagated apricot populations: two main gene pools in Tunisia evidenced by SSR markers and model-based Bayesian clustering. Genetica 138: 1023-1032

Cipriani G, Lot G, Huang W G, Marrazzo M T, Peterlunger E \& Testoline R (1999). AC/GT and AG/ CT microsatellite repeats in peach [Prunus persica (L) Batsch] isolation, characterization and crossspecies amplification in Prunus. Theoretical and Applied Genetics 99: 65-72

Dumanoğlu H, Bakır M, Ernim C \& Macit T (2018). Evaluation of wild apricot (Prunus armeniaca L.) genetic materials around Nevşehir province for late blooming, resistance to late spring frosts, large fruit size and late fruit maturity. The Final Report of Scientific Research and Technological Research Project (1140279). TUBITAK, Ankara

Ercisli S (2004). A short review of the fruit germplasm resources of Turkey. Genetic Resources and Crop Evolution 51: 419-435

Eroglu D \& Cakir B (2015). Molecular characterization of sweet cherry genotypes and rootstocks by using Prunus SSR sequences. Austin Journal of Biotechnology \& Bioengineering 2(3): 1044

Gupta P K, Balyan H S, Sharma P C \& Ramesh B (1996). Microsatellites in plants: a new class of molecular markers. Current Science 70: 45-54

Gürcan K, Öcal N, Yilmaz K U, Ullah S, Erdogan A \& Zengin Y (2015). Evaluation of Turkish apricot germplasm using SSR markers: Genetic diversity assessment and search for Plum pox virus resistance alleles. Scientia Horticulturae 193: 155-164

Hagen L S, Chaib J, Fady B, Decroocq V, Lambert J P \& Audergon J M (2004). Genomic and cDNA microsatellites from apricot (Prunus armeniaca L.). Molecular Ecology Notes 4: 742-745
Hormaza J I (2002). Molecular characterization and similarity relationships among apricot (Prunus armeniaca L.) genotypes using simple sequence repeats. Theoretical and Applied Genetics 104: 321328

Kostina KF (1969). The use of varietal resources of apricots for breeding. Trudy Nikitiskogo Botanicheskogo Sada 40: 45-63

Layne R E C, Bailey C H \& Hough L F (1996). Apricots. In: J Janick \& J N Moore (Eds.) Fruit Breeding: Tree and Tropical Fruits, Jhon Viley \& Sons, Inc. New York, pp.79-111

Lefort F, Lally M, Thompson D \& Douglas G C (1998). Morphological traits, microsatellite fingerprinting and genetic relatedness of a stand of elite oaks ( $Q$. robur L.) at Tuallynally, Ireland. Silvae Genetica 47: 5-6

Litt M \& Luty J A (1989). A hypervariable microsatellite revealed by in vitro amplification of a dinucleotide repeat within the cardiac muscle actin gene. The American Society of Human Genetics 44: 397-401

Liu H U, Liu J, Wang Z, Ma L Y, Wang S Q, Lin X G, Wu R L \& Pang X M (2013). Development and characterization of microsatellite markers in Prunus sibirica (Rosaceae). Applications in Plant Sciences 1(3): apps. 1200074

Liu K \& Muse S V (2005). PowerMarker: an integrated analysis environment for genetic marker analysis. Bioinformatics 21: 2128-2129

Messina R, Lain O, Marrazzo M T, Cipriani G \& Testolin $\mathrm{R}$ (2004). New set of microsatellite loci isolated in apricot. Moleculer Ecology Notes 4: 432-434

Mnejja M, Garcia-Mas J, Howad W \& Arus P (2005). Development and transportability across Prunus species of 42 polymorphic almond microsatellites. Molecular Ecology Notes 5: 531-535

Murathan Z T, Kafkas S, Asma B M \& Topçu H (2017). $S$ allele identification and genetic diversity analysis of apricot cultivars. The Journal of Horticultural Science and Biotechnology 92: 251-260

Nei M (1973). Analysis of gene diversity in subdivided populations. The Proceedings of the National Academy of Science 70: 3321-3323

Romero C, Pedryc A, Munoz V, Llacer G \& Badenes M L (2003). Genetic diversity of different apricot geographical groups determined by SSR markers. Genome 46: 244-252 
Ruthner S, Pedryc A, Krska B, Romero C \& Badenes M L (2006). Molecular characterization of apricot (Prunus armeniaca L.) cultivars using cross species SSR amplification with peach primers. International Journal of Horticultural Science 12: 53-57

Sanchez-Perez R, Ruiz D, Dicent F, Egea J \& MartínezGómez P (2005). Application of simple sequence repeat (SSR) markers in apricot breeding: molecular characterization, protection, and genetic relationships. Scientia Horticulturae 103: 305-315

Schuelke M (2000). An economic method for the fluorescent labelling of PCR fragments. Nature Biotechnology 18: 233-234

Tamura K, Dudley J, Nei M \& Kumar S (2007). MEGA4: Molecular Evolutionary Genetics Analysis (MEGA) software version 4.0. Molecular Biology and Evolution 24: 1596-1599

TUIK (2017). Number of wild apricot trees in Nevşehir. https://biruni.tuik.gov.tr/medas $/$ kn $=92 \&$ locale $=$ tr (Retrieved in 15.12.2017)

Ullah S, Aish M, Iqbal H, Hafeez R \& Muhammad Z H (2017). Genetic analysis of economically important apricot cultivars in Gilgit Baltistan based on SSR molecular markers. Romanian Biotechnological Letters 22: 12456-12463
Vavilov N I (1951). The Origin, variation, immunity and breeding of cultivated plants (Translated by S. K. Chestitee). Chronica Botonica 13: 1-366

Wang Z, Kang M, Liu H, Gao J, Zhang Z, Li Y, Wu R \& Pang X (2014). High-level genetic diversity and complex population structure of Siberian apricot (Prunus sibirica L.) in China as revealed by nuclear SSR markers. PLoS One 9, e87381. doi:10.1371/ journal.pone. 0087381

Wünsch A (2009). Cross-transferable polymorphic SSR loci in Prunus species. Scientia Horticulturae 120: 348-352

Yamamoto T, Mochida K, Imai T, Shi Y Z, Ogiwara T \& Hayashi T (2002). Microsatellite markers in peach [Prunus persica (L.) Batsch] derived from an enriched genomic and cDNA libraries. Molecular Ecology Notes 2: 298-301

Zhebentyayeva T N, Reighard G L, Gorina V M \& Abbott A G (2003). Simple sequence repeat (SSR) analysis for assessment of genetic variability in apricot germplasm. Theoretical and Applied Genetics 106: 435-444

Zhebentyayeva T, Ledbetter C A, Burgos L \& Llàcer G (2012). Apricot. In: M L Badenes \& P H Byrne (Eds.) Fruit Breeding, Springer, New York, pp. 415-458 